
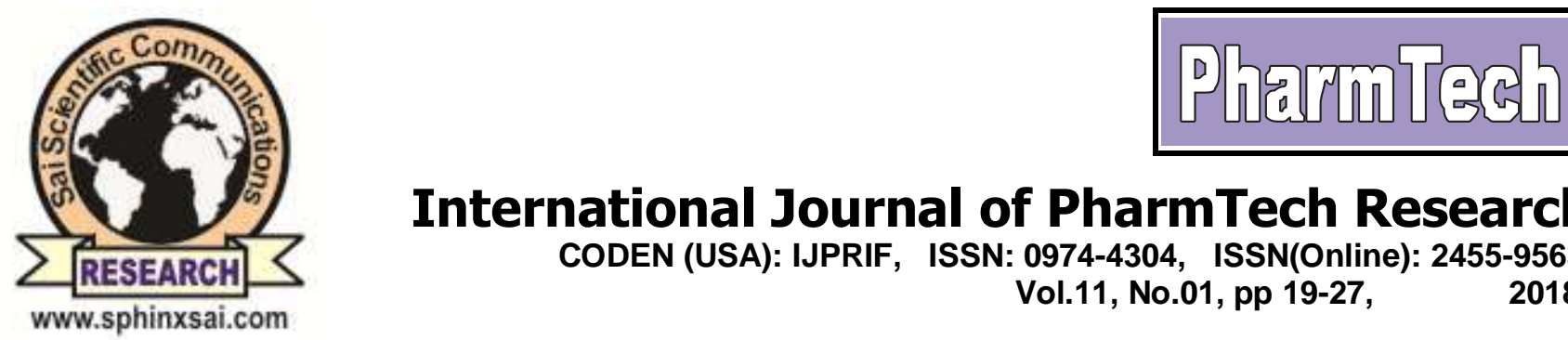

International Journal of PharmTech Research

CODEN (USA): IJPRIF, ISSN: 0974-4304, ISSN(Online): 2455-9563

Vol.11, No.01, pp 19-27,

2018

\title{
The Effect of Curcumin on Cognitive Dysfunction Through Cholinergic Pathway in Hippocampus, Frontal Cortex and Caspase 3 in Rat Shohami's Weight-Drop Model Traumatic Brain Injury
}

\author{
Amin Atiya Alnawajha ${ }^{1 *}$, Mulyohadi Ali ${ }^{2}$, Tommy A. Nazwar ${ }^{3}$ \\ ${ }^{1}$ Master of Biomedical Program, Medical Faculty, Brawijaya University, Malang, \\ Indonesia \\ ${ }^{2}$ Department of Pharmacology, Medical Faculty, Brawijaya University, Malang, \\ Indonesia \\ ${ }^{3}$ Department of Neurosurgery, Saiful Anwar Hospital, Medical Faculty, Brawijaya \\ University, Malang, Indonesia.
}

\begin{abstract}
TBI (traumatic brain injury) results in significant disability due to cognitive deficits mainly in attention, learning and memory and higher-order executive functions. In this study we examined the effect of Curcumin on cognitive dysfunction through increase ACH and decrease caspase 3 expression in hippocampus and cortex. Twenty five anaesthetized Wistar rats were subjected to TBI using Shohami weight-drop model. The ACH and caspase 3 activity was determined using immunohistochemistry in brain tissue slices after 5 days treatment of Curcumin at 3 different doses $50 \mathrm{mg} / \mathrm{kg}, 100 \mathrm{mg} / \mathrm{kg}, 200 \mathrm{mg} / \mathrm{kg}$ and examined cognitive function using MWM. Increase expression of ACH was observed in hippocampus and cortex of brain after administration of curcumin in TBI, also was observed decrease expression of caspase 3 and improvement of cognitive dysfunction. In addition to observation above we found that increase expression of $\mathrm{ACH}$ no significant difference between treatment group, while decrease expression of caspase 3 was significant with high dose, and leading to good outcome of cognitive dysfunction. From our result we suggest that after TBI administration of curcumin may improve cognitive dysfunction through mechanism involving increasing of expression of $\mathrm{ACH}$ and decreasing of expression of caspase 3 and therefore decreasing apoptosis.
\end{abstract}

Key words : Caspase 3, cholinergic, curcumin, TBI.

Introduction

Traumatic Brain Injury can causing brain damage include structural and/or physiological disruption of brain function ${ }^{1}$. One of the most important cognitive sequelae of traumatic brain injury (TBI) is memory impairment ${ }^{2}$. Memory dysfunction after TBI implicated the hippocampus as an important structure for memory function ${ }^{3}$.

International Journal of PharmTech Research, Vol.11, No.1, pp 19-27, (2018)

http://dx.doi.org/10.20902/IJPTR.2018.11103 
Primary and secondary injuries that occured in the brain are responsible for cognitive dysfunction following TBI. Many biochemical derangements that are responsible for secondary injury have been identified. These include glutamate excitotoxicity, disorder of cellular calcium homeostasis, increased free radical generation and lipid peroxidation, mitochondrial dysfunction, inflammation, apoptosis and diffuse axonal injury ${ }^{4,5}$.

Activation of caspase- 3 induced by two caspase initiator pathways: a caspase-8-mediated pathway activated through cell surface death receptors (extrinsic pathway). And a caspase-9-mediated pathway activated by signals from the mitochondria that lead to formation of an apoptosomal complex ${ }^{6}$. TBI lead to reduction in cholinergic function, which reflected by decreasing of $\mathrm{ACH}$ in hypocampus. The cholinergic pathway responsible for process of cognition arousal and attention, therefore any change in this pathway may result in cognitive deficit after $\mathrm{TBI}^{7}$. Curcumin is the active ingredient of the dietary spice turmeric and used for medicinal purposes for thousands of years. Curcumin has been shown to have anti-inflammatory, anti-oxidant, pro-apoptotic, chemotherapeutic, anti-proliferative, wound healing, anti-nociceptive ${ }^{8}$.

The aim of this study to observe its effect of curcumin on improving cognitive function (memory) by inhibition caspase 3 and increasing level of $\mathrm{ACH}$ in traumatic brain injury, and the potency of curcumin to protect brain from damage.

\section{Experimental}

This study is true experimental design, in this study 25 male Wistar rat where divided into 5 group consisting of 5 rat, including control negative $(\mathrm{C}-)$, control positive $(\mathrm{C}+), \mathrm{T} 1, \mathrm{~T} 2$, T3. Where control negative group without trauma and treatment, control positive group with TBI only, T1 with traumatic brain injury, which was administered intraperitoneal $50 \mathrm{mg} / \mathrm{kg}$ body weight curcumin, T2 with TBI which was administered intraperitoneal $100 \mathrm{mg} / \mathrm{kg}$ body weight curcumin, T3 with TBI which was administered intraperitoneal 200 $\mathrm{mg} / \mathrm{kg}$ body weight curcumin.

\section{Experimental Traumatic Brain Injury Model}

Shohami's weight drop model used, the rats were anesthetized, midline incision over the skull performed, the skin retracted, and the skull exposed to locate the area of impact, then $3 \mathrm{~mm}$ diameter plastic tip of a metal rod weighting $35 \mathrm{~g}$ gently drop from $100 \mathrm{~cm}$ height onto exposed skull at the left hemisphere $2 \mathrm{~mm}$ lateral from the midline and $2 \mathrm{~mm}$ back from the coronal suture ${ }^{9,10}$. To evaluation of neurological motor function deficit in rat after TBI was used Neurological Severity Score (NSS) (Table 1) ${ }^{\mathbf{1 1}}$.

\section{Cognitive Testing}

The test was performed in Moris Water Mase (MWM), round pool contain water with $26^{\circ} \mathrm{C}$ and place platform in center of the pool .The rat trained in the water maze with 12 consecutive trials per day for 3 days. First, put the rat on the platform for twenty seconds. The water maze has 4 starting positions: north, south, east, or west. Take the animal to one of these positions, and rat swim and search for the platform for a maximum of 60 seconds, when mice reaches the platform, stop the timer, and record the time ${ }^{\mathbf{1 1}}$.

\section{Immunohistochemistry}

After finished of treatment, the rats sacrificed, the brain tissue removed and put in formalin $10 \%$ not more than 24 hours, the following steps are dehydration, clearing, embedding, block in paraffin. The tissue was cut with thickness of $6 \mu \mathrm{m}$ using microtome. Immunohistochemistry staining was done to see caspase 3 and $\mathrm{ACH}$ distribution in hippocampus and cortex. At first slide incubate at $60 \mathrm{C}$ for 1 hour, wash specimen with $\mathrm{dH}_{2} \mathrm{O} 3$ time for 5 minute apply $3 \% \mathrm{H}_{2} \mathrm{O}_{2}$ in methanol for 15 minute at room temperature, then wash specimen 3 time of PBS for 5 minute. Dilution (1:1000) primer antibody anti caspase 3 in blocking buffer, then incubate overnight at $40 \mathrm{C}$. Wash specimen 3 time of PBS for 5 minute. Apply secondary antibody to specimen for hour at room temperature, wash specimen for 3 time of PBS for 5 minute. Apply SA-HRP to specimen for 45 minute at room temperature, then wash 3 time of PBS for 5 minute, and wash once in $\mathrm{H}_{2} 0$ for 1 minute. Dilute DAB

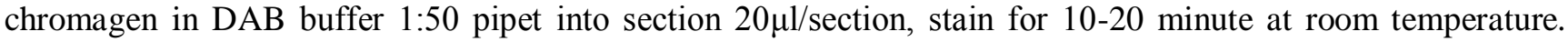
Wash specimen 3 time of $\mathrm{dH}_{2} \mathrm{O}$ for 5 minute. Apply Mayer haematoxylin in tap water to specimen for 5 minute, 
wait until specimen to dry. Immunohistochemistry staining were examined by Olympus microscope, by observing brown colour seen in 20 field of views with magnification of 1000x.

\section{Statistical Analysis}

All data was subjected to statistical analysis. We analyzed our data using One-Way ANOVA test followed by Post-Hoc test (LSD), a significant $\mathrm{p}<0.05$, to assess the comparison test followed by Post-Hoc test (LSD), a significant $\mathrm{p}<0.05$, to assess the comparison between groups. We considered $<0.05$ as significant.

\section{Results}

\section{Expression of $\mathrm{ACH}$ in Hippocampus}

As shown in Figure 1 that control positive group expression of ACH in hippocampus after TBI decreased compared with treatment group, but expression of ACH between treatment group was more expressed in T3. Positive expression noted with brown color in hippocampus.
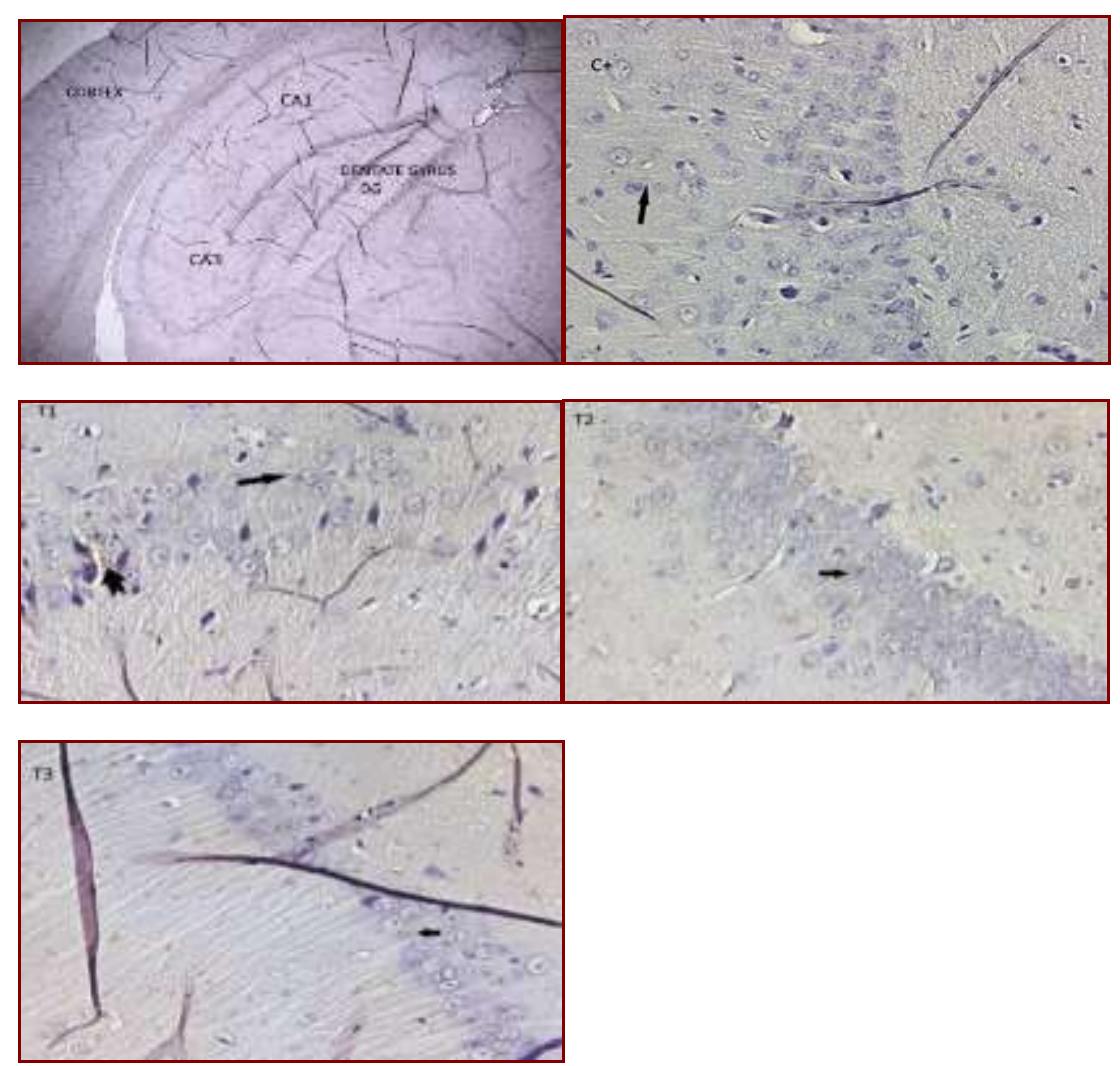

Figure 1. The effects of TBI in the distribution of $\mathrm{ACH}$ on hippocampus. Immunohystochemistry was done by DAB staining (brown color). Compared to the control group (C+,-), ACH expression decrease after TBI (C+), while ACH expression in treatment groups increased (T1, 2, 3). Arrows indicate expression of $\mathrm{ACH}$.

Based on Figure 2 below shows that giving curcumin at $100 \mathrm{mg} / \mathrm{kg}$ (T2) can increase the average of expression of $\mathrm{ACH}$ in hippocampus rather than the provision of curcumin at a dose at $200 \mathrm{mg} / \mathrm{kg}$ (T3), so dose $100 \mathrm{mg} / \mathrm{kg}$ (T2) was more effective to increase expression of ACH in hippocampus. However, the provision of curcumin at $200 \mathrm{mg} / \mathrm{kg}$ (T3) can increase the average expression of $\mathrm{ACH}$ in hippocampus better than dose of 50 $\mathrm{mg} / \mathrm{kg}$ (T1), so T3 was more effective to increase expression of $\mathrm{ACH}$ in hippocampus than T1. But the average of expression of $\mathrm{ACH}$ in hippocampus TBI in granting curcumin at T1 was higher than the positive control group, so this group is effective to increase expression of $\mathrm{ACH}$ in hippocampus. 


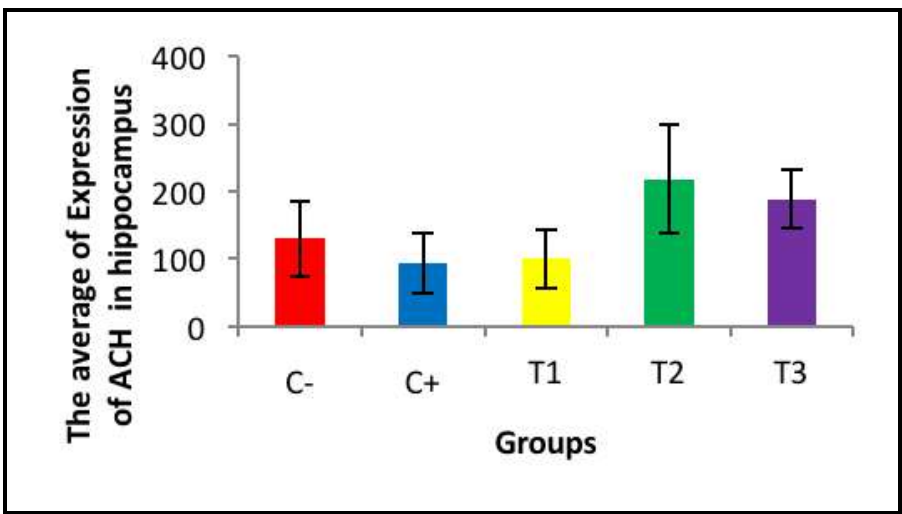

Figure 2. Expression of $\mathrm{ACH}$ in hippocampus at each dose of curcumin. (C-) control negative, $(\mathrm{C}+)$ control positive, T1 $(50 \mathrm{mg} / \mathrm{kg}$ curcumin $), T 2(100 \mathrm{mg} / \mathrm{kg}), \mathrm{T} 3(200 \mathrm{mg} / \mathrm{kg})$

\section{Expression of $\mathrm{ACH}$ in Cortex}

In Figure 3 shown that in control positive group animal no observable expression of $\mathrm{ACH}$ in the cortex of brain, but in other group treatment of animal was observable expression with different degree, as we observed in group T3 at dose of curcumin $200 \mathrm{mg} / \mathrm{kg}$ more expression compared with others.
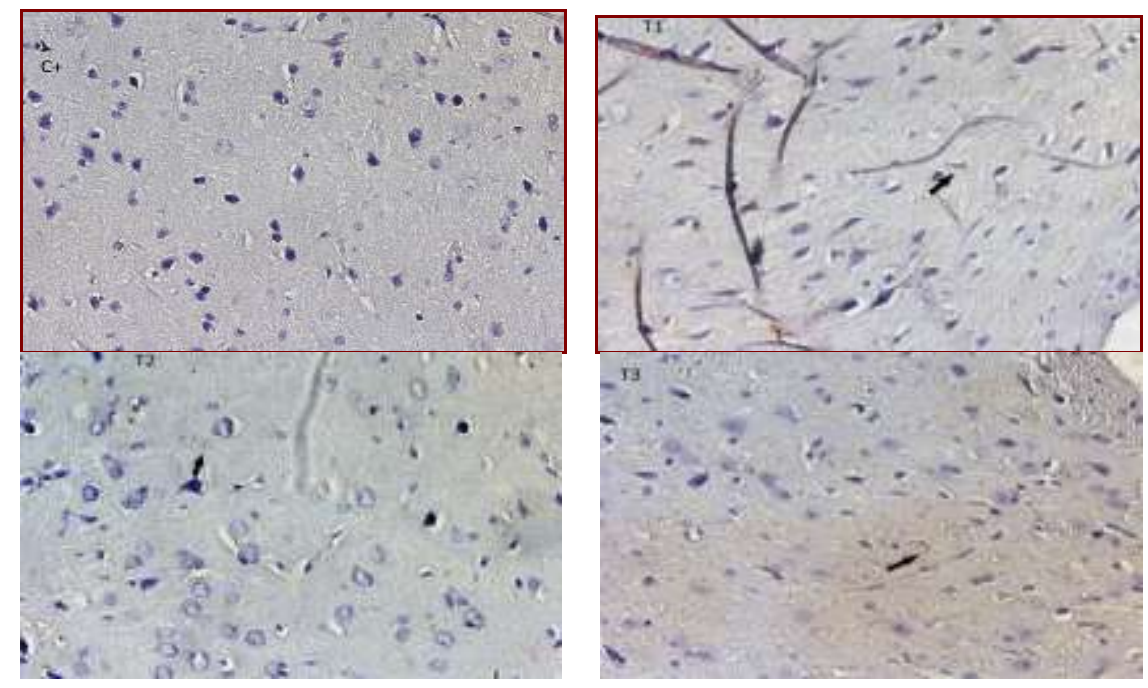

Figure 3. The effects of TBI in distribution of $\mathrm{ACH}$ in the cortex of brain tissue. The figure shows the immunohistochemistry staining visualized by DAB (brown). Compared to the control group (C+,-), ACH expression decrease after TBI $(\mathrm{C}+)$, while $\mathrm{ACH}$ expression in treatment groups increased $(\mathrm{T} 1,2,3)$. Arrow indicate expression $\mathrm{ACH}$ in cortex.

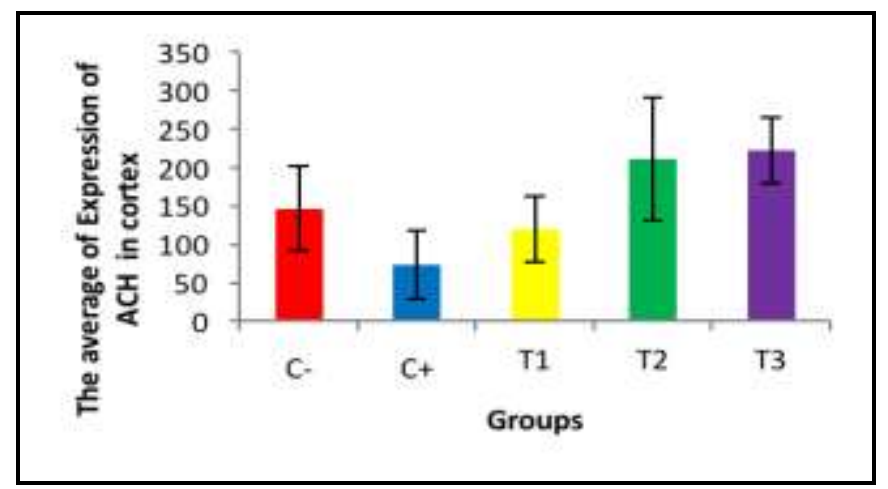

Figure 4. Expression of $\mathrm{ACH}$ in cortex at each dose curcumin $(\mathrm{C}$-) control negative, $(\mathrm{C}+)$ control positive, T1 (50 mg/kg curcumin), T2 (100 mg/kg),T3 (200 mg/kg) 
Based on Figure 4 below shows that giving curcumin at dose $200 \mathrm{mg}$ (T3) can increase the average of expression of $\mathrm{ACH}$ in cortex rather than the provision of curcumin at a dose at dose $0 \mathrm{f} 100 \mathrm{mg}$ (T2), so T3 was more effective to increase expression of $\mathrm{ACH}$ in cortex than T2. However, the provision of curcumin at $\mathrm{T} 2$ can increase the average expression of $\mathrm{ACH}$ in cortex better than dose $50 \mathrm{mg}$ (T1), so T2 was more effective to increase Expression of ACH in cortex than T1. But the average of expression of ACH in cortex TBI in granting curcumin at T1 was higher than the positive control group, so this group is effective to increase expression of $\mathrm{ACH}$ in cortex.

From data above after we administered different concentrations of curcumin $(50,100$, or $200 \mathrm{mg} / \mathrm{kg})$ to rats post-TBI for 5 days, and examine $\mathrm{ACH}$ expression in hippocampus and cortex. The administration of 3 doses curcumin did not significantly increase expression of ACH in hippocampus compared with TBI alone (P $>0.05$ ). In contrast, increase expression of $\mathrm{ACH}$ in the cortex of brain at $100 \mathrm{mg} / \mathrm{kg}$ or $200 \mathrm{mg} / \mathrm{kg}$ curcumin significantly increase $\mathrm{ACH}$ expression $(\mathrm{P}<0.05)$.

\section{Expression of Caspase 3 in Hippocampus:}

As shown in Figure 5 in control positive group that expression of caspase 3 in hippocampus after TBI, was observable and more intense compared with other group, where in group treatment T1, T2, T3 also observed that more dose of curcumin, less intense of expression of caspase 3 in hippocampus.

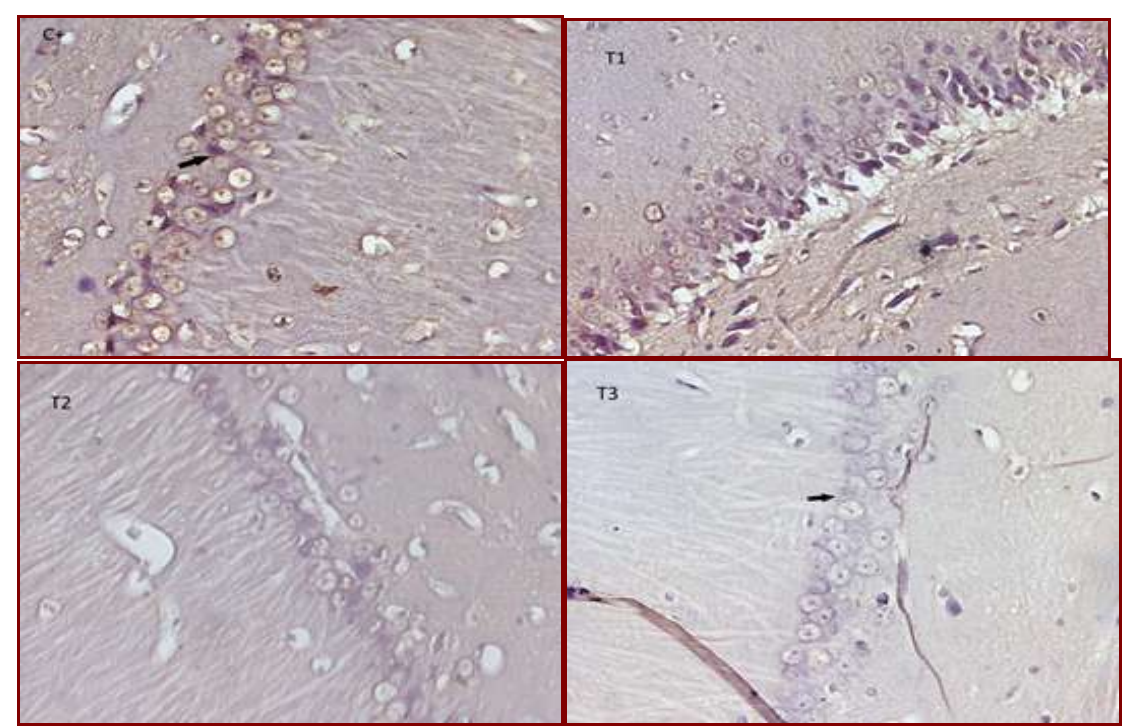

Figure 5. The effects of TBI in distribution of caspase 3 in Hippocampus. The figure shows the immunohistochemistry staining of visualized by DAB (brown). Compared to the control group (C+,-), Caspase 3 expression increase after TBI $(\mathrm{C}+)$, while caspase 3 expression in treatment groups increased $(\mathrm{T} 1,2,3)$.

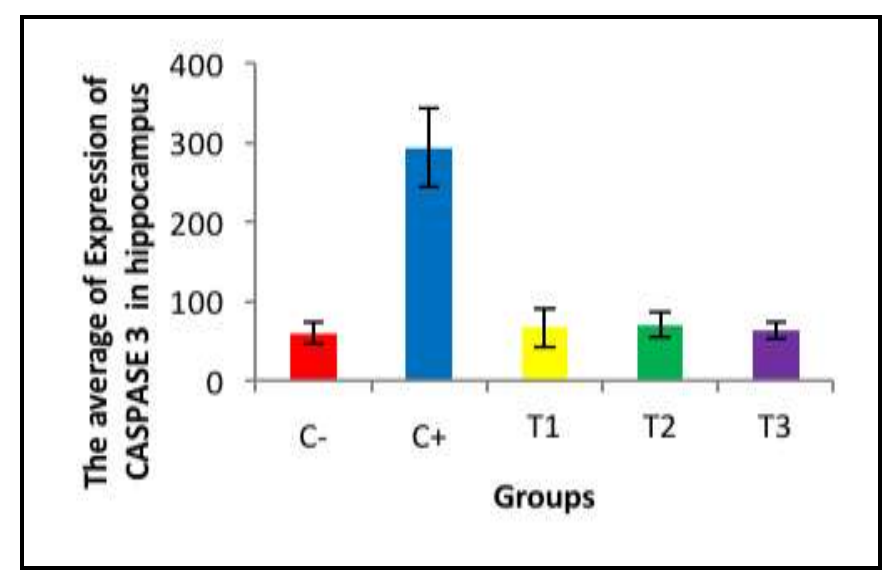

Figure 6. Expression of caspase 3 in hippocampus at each dose curcumin (C-) control negative, $(\mathrm{C}+)$ control positive, T1 (50 mg/kg curcumin), T2 (100 mg/kg), T3 (200 mg/kg) 
Based on Figure 6 above shows that giving curcumin at $200 \mathrm{mg}$ (T3) can decrease the average of expression of caspase 3 in hippocampus rather than the provision of curcumin at a lower dose at $50 \mathrm{mg}$ (T1) and $100 \mathrm{mg}$ T2, so T3 was more effective to decrease expression of caspase 3 in hippocampus than T1 and T2. However, the provision of curcumin at T2 can decrease the average expression of caspase 3 in hippocampus better than $50 \mathrm{mg}$ (T1), so T2 was more effective to decrease expression of caspase 3 in hippocampus than T1.

\section{Expression of Caspase 3 in Cortex}

As shown in Figure 7 in control positive group that expression of caspase 3 in cortex of brain after TBI and was observable and more intense compared with other group, where in group treatment T1, T2, T3 also observed that more dose of curcumin, less intense of expression of caspase 3 in hippocampus thus in group T3 the expression of caspase 3 less intense compared with group T1 and T2.
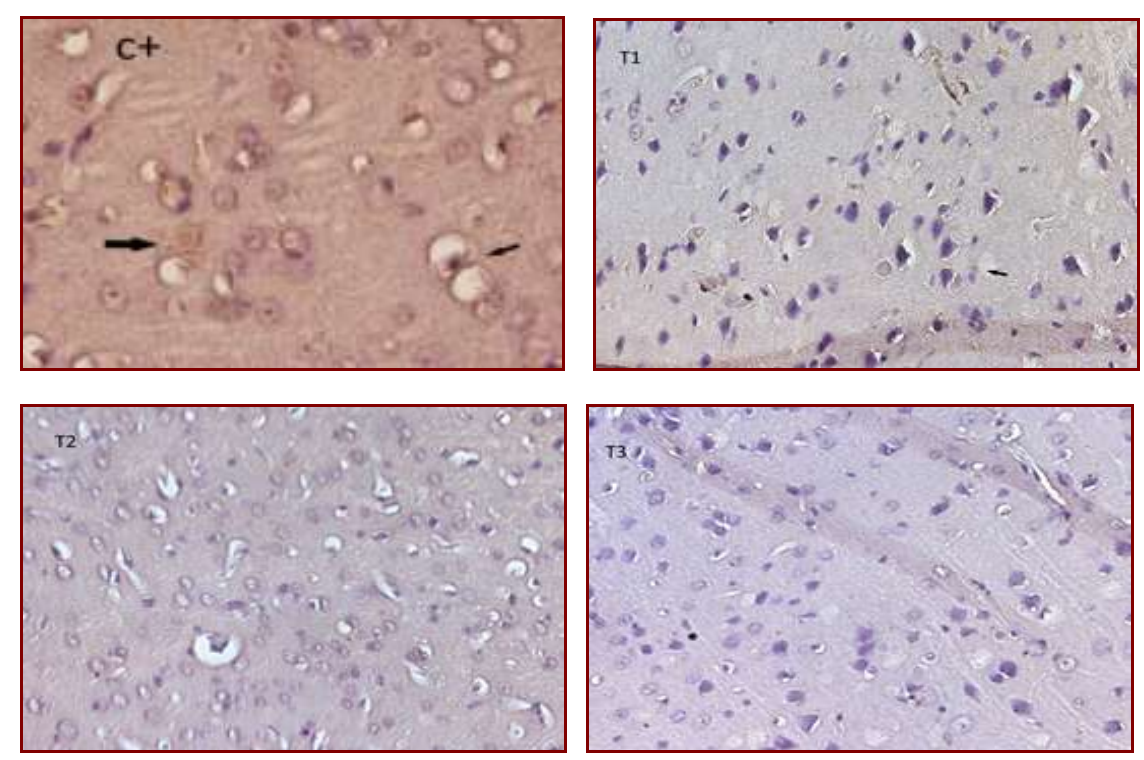

Figure 7. The effects of TBI in distribution of caspase 3 in cortex. The figure shows the immunohistochemistry staining of visualized by DAB (brown). Compared to the control group (C+,-), caspase 3 expression increase after TBI $(\mathrm{C}+)$, while caspase 3 expression in treatment groups increased $(\mathrm{T} 1,2,3)$.

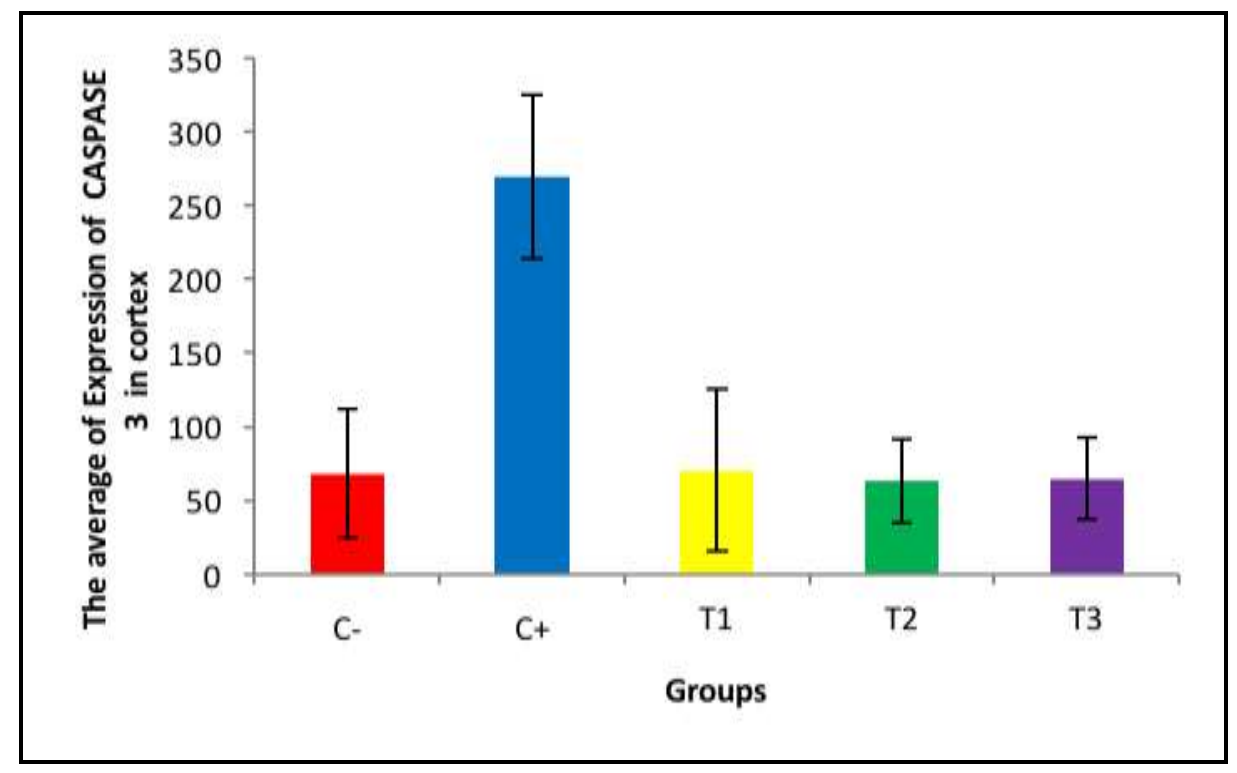

Figure 8. Expression of caspase 3 in cortex at each dose of curcumin. $(\mathrm{C}-)$ control negative, $(\mathrm{C}+)$ control positive, T1 (50 mg/kg curcumin), T2 (100 mg/kg), T3 (200 mg/kg) 
Based on above giving curcumin at dose of $200 \mathrm{mg}$ (T3) can decrease the average of expression of caspase 3 in cortex rather than the provision of curcumin at a dose at $100 \mathrm{mg}$ (T2), so T3 was more effective to decrease expression of caspase 3 in cortex than T2. However, the provision of curcumin at T2 can decrease the average Expression of caspase 3 in cortex better than $50 \mathrm{mg}$ (T1), so T2 was more effective to decrease expression of caspase 3 in cortex than $\mathrm{T} 1$.

From data above after we administered different concentrations of curcumin $(50,100,200 \mathrm{mg} / \mathrm{kg}$ ) to rats post-TBI for 5 days, and examine caspase 3 expression in hippocampus and cortex. The administration of 3 doses curcumin was significantly decrease expression of caspase 3 in hippocampus compared with TBI alone (P $>0.05$ ) also decrease expression of caspase in the cortex of brain at 3 different doses of curcumin was significantly decreae caspase 3 expression $(\mathrm{P}<0.05)$.

\section{MWM Pre-Experimental and Post-Experimental TBI Post-treatment}

From data on Figure 9 shows that giving curcumin at $200 \mathrm{mg}$ (T3) can decrease of MWM PostExperimental TBI Post-treatment rather than the provision of curcumin at a lower dose at $100 \mathrm{mg}$ (T2), so dose T3 was more effective to decrease MWM Post-Experimental TBI Post-treatment than T2. However, the provision of curcumin at $100 \mathrm{mg}$ (T2) can decrease the average MWM Post-Experimental TBI Post-treatment better than $50 \mathrm{mg}$ (T1), so dose T2 was more effective to decrease MWM Post-Experimental TBI Post treatment than dose $\mathrm{T} 1$.

A.

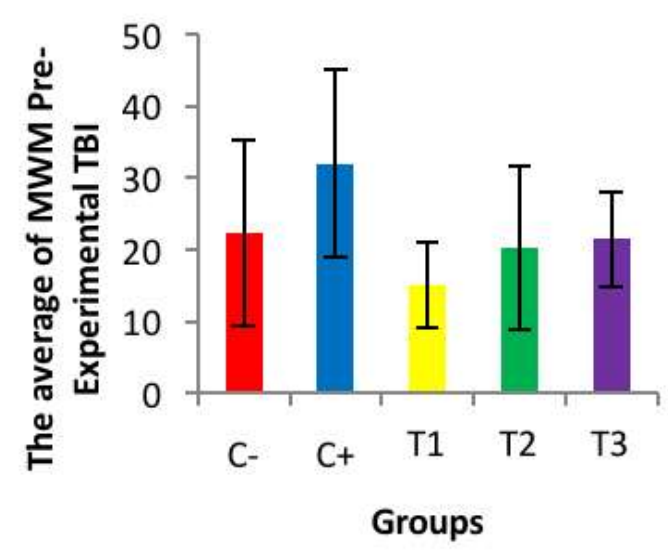

B.

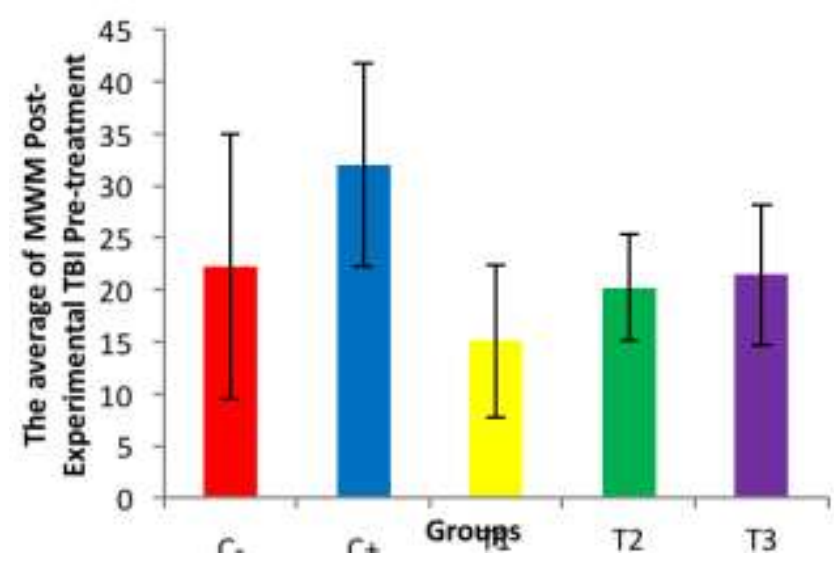

C.

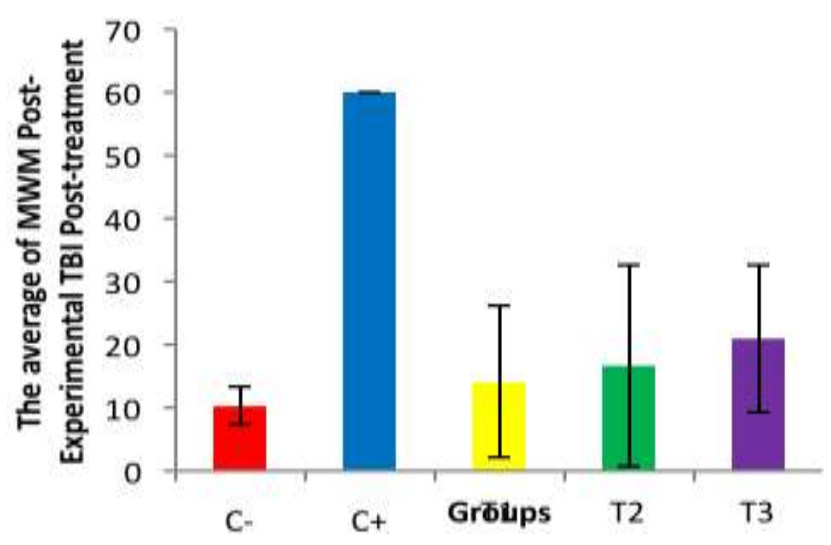

Figure 9. MWM Post-experimental TBI Post-treatment at each dose curcumin 


\section{Discussions}

\section{Effect of Curcumin on ACH and Cognitive Dysfunction in TBI}

In this study, we used curcumin to observe its effect in rats post TBI to improve cognitive dysfunction through increase ACH and decrease caspase 3 which implicated in this dysfunction, TBI can result in cognitive deficits mainly in attention, learning and memory ${ }^{4}$. Outcome of TBI determine by different stage of injury: primary and secondry damage, where primary damage ocurre at moment of trauma, while secondary damage is consecutive processes that begin at moment of injury ${ }^{12}$. After TBI can occur reduction in cholinergic function by decreasing of $\mathrm{ACH}$ in hypocampus that responsible for cognition ${ }^{13}$.

In this study used curcumin in 3 various dose, to investigate about the effect of curcumin on $\mathrm{ACH}$, caspase 3 and cognitive dysfunction after TBI. In our study after administration curcumin in various doses, the result showed that curcumin can increased expression of $\mathrm{ACH}$ in hippocampus. Giving curcumin at dose of 100 $\mathrm{mg} / \mathrm{kg}$ can increase expression of $\mathrm{ACH}$ in hippocampus rather than the provision of curcumin at a dose at 200 $\mathrm{mg} / \mathrm{kg}$, so dose of $100 \mathrm{mg} / \mathrm{kg}$ was effective to increase expression of $\mathrm{ACH}$ in hippocampus than $200 \mathrm{mg} / \mathrm{kg}$. However, the provision of curcumin at $200 \mathrm{mg} / \mathrm{kg}$ can increase expression of $\mathrm{ACH}$ in hippocampus better than $50 \mathrm{mg} / \mathrm{kg}$. While in cortex giving curcumin at dose of $200 \mathrm{mg} / \mathrm{kg}$ can increase of expression of ACH in cortex rather than the provision of curcumin at a dose at $100 \mathrm{mg} / \mathrm{kg}$, thus the dose of $200 \mathrm{mg} / \mathrm{kg}$ was more effective to increase expression of ACH in cortex than $100 \mathrm{mg} / \mathrm{kg}$. However, the provision of curcumin at $100 \mathrm{mg} / \mathrm{kg}$ can increase Expression of ACH in cortex better than dose of $50 \mathrm{mg} / \mathrm{kg}$, thus dose $100 \mathrm{mg} / \mathrm{kg}$ was more effective to increase expression of $\mathrm{ACH}$ in cortex than $50 \mathrm{mg} / \mathrm{kg}$. As mention before $\mathrm{ACH}$ play important role in cognitive function, but in TBI can causing cholinergic dysfuntion leading to deficits in learing and memory due to chlolinergic neuronal loss in many area of brain ${ }^{14}$. The mechanism how cholinergic neuron susceptible to trauma unknow, but it is suggested that disruption in nerve growth factor production and transport to these neurons, another suggestion that these neurons susceptible to excitotoxic of potassium and glutamate which release after TBI ${ }^{15}$. From this point it is suggested that curcumin play role in prevent or neuroprotection in TBI. There was study ${ }^{16}$ about dietry curcumin in TBI on oxidative stress, synaptic plasticity, and cognition, and this study reported that curcumin has antioxidant effect and can reduce oxidative damage on synaptic plasiticity and cognitive function.

\section{Effect of Curcumin on Caspase 3 and Cognitive Dysfunction in TBI}

Curcumin at dose $200 \mathrm{mg} / \mathrm{kg}$ can decrease expression of caspase 3 in hippocampus rather than the provision of Curcumin at a lower dose at dose $50 \mathrm{mg} / \mathrm{kg}$ and $100 \mathrm{mg} / \mathrm{kg}$, thus dose $200 \mathrm{mg} / \mathrm{kg}$ was more effective to decrease expression of CASPASE 3 in hippocampus. curcumin at 3 different doses $50 \mathrm{mg} / \mathrm{kg}, 100$ $\mathrm{mg} / \mathrm{kg}$ and $200 \mathrm{mg} / \mathrm{kg}$ can decrease expression of caspase 3 in cortex. In this study we found decreasing expression of caspase 3 in hippocampus more at higher dose, while decreasing expression of caspase 3 in cortex with different doses, we found effect is the same. Where apoptosis increase due to oxidative stress that induce production free radical, hydrogen peroxide, nitric oxide in TBI. Excessive production of reactive oxygen species due to deficite of endogenous antioxidant system, causing immediate cells death and early or late apoptosis $^{\mathbf{1 2}}$, and this data suggest that curcumin act through its antioxidant effect .

\section{Correlation between ACH, Caspase 3 and Cognitive Dysfunction}

At first there is correlation between doses of curcumin and expression of $\mathrm{ACH}$ and expression of caspase 3 in cortex and hippocampus. The expression of ACH in hippocampus and cortex have coefficient of correlation equals to 0.503 and 0,690 respectively, which shows us relation quite strong. Also correlation between doses of curcumin and expression of caspase 3 in hippocampus and cortex and have coefficient of correlation equals to $-0,669$ and $-0,636$ respectively, and it's quite strong. That mean the increase of doses of curcumin will be followed increase of expression of $\mathrm{ACH}$ in hippocampus and cortex, and will be decrease of expression of caspase 3 in hippocampus and cortex, and therefore will improve cognitive function. 


\section{Conclusion}

Our finding suggest that curcumin administration may improve cognitive dysfunction outcome through mechanism increase $\mathrm{ACH}$, and decrease activity of caspase 3 (neuronal apoptosis) at dose high as $200 \mathrm{mg} / \mathrm{kg}$ of curcumin.

\section{Acknowledgement} study.

Author thank to Medical Faculty, Brawijaya University, Malang, East Java, Indonesia for facilitating this

\section{Conflict of Interest}

Author declare no conflicts of interest

\section{References}

1. Vos, P. E., \& Arrastia, R. D. Traumatic Brain Injury, First Edition, John Wiley \& Sons, India, 2015, 91.

2. Serra-Grabulosa, J. M., Junque, C., Verger, K., Pineda, S., Maneru, C., \& Mercader, J. M. (2005).

Cerebral correlates of declarative memory dysfunctions in early traumatic brain injury. Journal of

Neurology, Neurosurgery, and Psychiatry, 76(1), 129-131. doi:10.1136/jnnp.2004.027631.

3. Hicks, R. R., Smith, D. H., Lowenstein, D. H., Marie, R. S., \& McINTOSH, T. K. (1993). Mild experimental brain injury in the rat induces cognitive deficits associated with regional neuronal loss in the hippocampus. Journal of Neurotrauma, 10(4), 405-414. doi:10.1089/neu.1993.10.405.

4. Walker, K. R., \& Tesco, G. (2013). Molecular mechanisms of cognitive dysfunction following traumatic brain injury. Frontiers in Aging Neuroscience, 5, 29. doi:10.3389/fnagi.2013.00029.

5. Xiong, Y., Mahmood, A., \& Chopp, M. (2013). Animal models of traumatic brain injury. Nature Reviews. Neuroscience, 14(2), 128-142. doi:10.1038/nrn3407.

6. Knoblach, S. M., Nikolaeva, M., Huang, X., Fan, L., Krajewski, S., Reed, J. C., \& Faden, A. I. (2002). Multiple caspases are activated after traumatic brain injury: evidence for involvement in functional outcome. Journal of Neurotrauma, 19(10), 1155-1170. doi:10.1089/08977150260337967.

7. Donat, C. K., Schuhmann, M. U., Voigt, C., Nieber, K., Schliebs, R., \& Brust, P. (2007). Alterations of acetylcholinesterase activity after traumatic brain injury in rats. Brain Injury : [BI], 21(10), 1031-1037. doi:10.1080/02699050701630359.

8. Gupta, S. C., Patchva, S., Koh, W., \& Aggarwal, B. B. (2012). Discovery of curcumin, a component of the golden Ssice, and its miraculous biological activities. Clinical and Experimental Pharmacology \& Physiology, 39(3), 283-299. doi:10.1111/j.1440-1681.2011.05648.x.

9. Khalin, I., Jamari, N. L. A., Abdul Razak, N. B., Hasain, Z. B., Mohd Nor, M. A., Zainudin, M. H. A., \& Alyautdin, R. (2016). A mouse model of weight-drop closed head injury: emphasis on cognitive and neurological deficiency. Neural Regeneration Research, 11(4), 630-635. doi:10.4103/1673-5374.180749.

10. Kobeissy, F., Dixon, E., Hayes, R., \& Mondello, S. Injury Models of the Central Nervous System Methods and Protocols, Springer Nature, New York, 2016, 194-198.

11. Nunes, J. (2008). Morris water maze experiment. Journal of Visualized Experiments, 19, 897.

12. Werner, C., \& Engelhard, K. (2007). Pathophysiology of traumatic brain injury. British Journal of Anaesthesia, 99(1), 4-9. doi:10.1093/bja/aem131.

13. Ciallella, J. R., Yan, H. Q., Ma, X., Wolfson, B. M., Marion, D. W., DeKosky, S. T., \& Dixon, C. E. (1998). Chronic effects of traumatic brain injury on hippocampal vesicular acetylcholine transport and M2 muscarinic receptor protein in rats. Experimental Neurology, 152(1), 11-19. doi:10.1006/exnr.1998.6831.

14. Shin, S. S., \& Dixon, C. E. (2015). Alterations in cholinergic pathways and therapeutic strategies targeting cholinergic system after traumatic brain injury. Journal of Neurotrauma, 32(19), 1429-1440. doi:10.1089/neu.2014.3445.

15. Schmidt, H. R., \& Grady, M. (1995). Loss of forebrain cholinergic neurons following fluid percussion injury: implications for cognitive impairment in closed head injury. Journal of Neurosurgery, 83(3), 496502. doi:10.3171/jns.1995.83.3.0496.

16. Wu, A., Ying, Z., \& Gomez-Pinilla, F. (2006). Dietary curcumin counteracts the outcome of traumatic brain injury on oxidative stress, synaptic plasticity, and cognition. Experimental Neurology, 197(2), 309317. doi:10.1016/j.expneurol.2005.09.004. 